\title{
Trivium
}

Revue franco-allemande de sciences humaines et sociales - Deutsch-französische Zeitschrift für Geistesund Sozialwissenschaften

$6 \mid 2010$

Esthétique et science de l'art

\section{Systématique et histoire des arts [1914]}

\section{Max Dessoir}

Traducteur : Françoise Joly

\section{(2) OpenEdition}

\section{Journals}

Édition électronique

URL : http://journals.openedition.org/trivium/3636

DOI : 10.4000/trivium.3636

ISSN : 1963-1820

Éditeur

Les éditions de la Maison des sciences de l'Homme

\section{Référence électronique}

Max Dessoir, «Systématique et histoire des arts [1914] », Trivium [En ligne], 6 | 2010, mis en ligne le 05 mai 2010, consulté le 08 septembre 2020. URL : http://journals.openedition.org/trivium/3636 ; DOI : https://doi.org/10.4000/trivium.3636

Ce document a été généré automatiquement le 8 septembre 2020

\section{(c) (i) () $\Theta$}

Les contenus des la revue Trivium sont mis à disposition selon les termes de la Licence Creative Commons Attribution - Pas d'Utilisation Commerciale - Pas de Modification 4.0 International. 


\section{Systématique et histoire des $\operatorname{arts}^{1}$ [1914]}

\section{Max Dessoir}

Traduction : Françoise Joly

1 L'esthétique a grandi dans les bras maternels de la philosophie. Mais la philosophie a toujours le droit de se porter vers l'esthétique sans perdre pour autant de son essence : le philosophe se tournant de tous les côtés pour trouver la signification et le fondement de ce qui est donné n'a pas besoin de détourner son regard des faits esthétiques. S'il veut appréhender en toute rigueur conceptuelle ce qui est et se manifeste en tant que beau, laid, sublime, etc., il doit pouvoir rapporter des constats empiriques (comme par exemple le caractère satisfaisant de certains couples de couleurs) à un concept plus général (par exemple celui d'harmonie), et rapporter ce dernier à son tour à une attitude intellectuelle plus large (disons, celle de la pure contemplation gratuite). C'est par cette réduction progressive aux principes les plus généraux que peuvent apparaître les relations [Zusammenhang] qu'entretiennent entre elles les connaissances singulières, ces dernières pouvant souvent prendre alors un nouveau sens. Grâce à cette méthode, on peut en particulier espérer saisir dans leur dernier fondement le caractère différent des grandes formes de culture que sont la religion, la science et l'art. Si des forces particulières sont à l'œuvre dans chacun de ces trois grands domaines, si des attitudes intellectuelles différentes constituent le préalable à ces formes de culture, il est sans doute aussi possible de définir la fonction rationnelle à travers laquelle l'esprit humain construit le champ de valeur esthétique. Si l'on pose que, dans le plaisir et la création artistiques, le donné est circonscrit et mis en forme par la fonction intellectuelle de la pure contemplation, non seulement l'attitude esthétique s'avère distincte des attitudes religieuse et scientifique mais, en outre, le trait fondamental qui traverse toute la vie esthétique se voit ainsi qualifié.

2 Pourtant, ce principe rationnel supérieur ne donne pas davantage qu'une définition plus précise de ce qu'il est. Certes, il permet sans doute d'éclairer d'un jour nouveau les faits artistiques, mais ces derniers n'en découlent pas entièrement. Les philosophes ont l'habitude d'échapper à cette perplexité en passant d'un raisonnement universellement 
applicable à l'expression d'un goût et d'une riche expérience personnels. Tout grand système comporte un point sur lequel, en se rapprochant toujours plus avant du singulier, la théorie objective se voit remplacée par une profession de foi subjective. Plus la personnalité est forte, plus son tact artistique est subtil et plus le passage du démontrable au vécu se fait imperceptible. Mais la fracture ne manque jamais d'être là.

Il y a contre cet état de choses, me semble-t-il, un seul moyen admissible : ce qui est ne prend pas une signification esthétique uniquement à partir du genre d'observation à laquelle il est soumis. C'est bien plutôt que certaines formes du réel répondent à cette activité et à cette finalité de la vie intellectuelle. Les choses ou les processus de la nature, de la culture et de l'art ont des caractéristiques objectives à travers lesquelles ils se distinguent des objets non esthétiques: ils sont, par exemple, "détachés du contexte réel », « imagés » [bildhaft], « construits par synthèse dans la tension entre un moment d'exaltation et un moment de satisfaction ». Il y a dans la structure même des objets esthétiques une nécessité qui préside à leur conformation esthétique. C'est surtout vrai pour la distinction, qui ne serait pas compréhensible autrement, entre les différents arts. La spécificité de la musique est manifestement en partie fondée sur la contrainte qui vient des sons, et qui exige de les combiner pour qu'ils résonnent ensemble simultanément, tandis que la peinture s'enracine dans la qualité différente des couleurs qui fait qu'elles ne peuvent être combinées que côte à côte dans l'espace. C'est ici sans aucun doute la matière de ce qui doit être réuni qui fait valoir sa particularité. C'est pourquoi l'esthétique philosophique est forcée de reconnaitre des lois qui, certes, sont de l'ordre de la raison, mais jamais seulement de la raison humaine. En passant à un tel objectivisme - et c'est ce qu'elle semble faire aujourd'hui elle acquiert, comme on le voit aisément, une fécondité accrue pour les sciences plus concrètes des différents arts.

4 En effet, la masse principale de la matière comportant de telles exigences objectives est présente dans les œuvres d'art, puisque l'art est une occasion privilégiée où peut intervenir l'attitude esthétique. Or, chaque œuvre d'art appelle d'une part à être appréciée selon son appartenance à un genre, par exemple en tant qu'œuvre poétique, ou pour prendre un exemple précis, dramatique, et demande d'autre part qu'y soit appréhendé avec force l'individualité d'un homme, d'un peuple, d'une époque qui opère en elle. Le particulier auquel nous sommes confrontés est donc dans l'art d'une nature en partie objective, en partie historique, et cela signifie qu'en partant d'un cas particulier on peut s'élever à un niveau supérieur de concepts généraux aussi bien systématiques qu'historiques. La connaissance du particulier n'est possible que s'il est inclus dans un contexte qui fasse sens - mais ce contexte doit-il d'abord être recherché dans les lois de la composition artistique ou dans l'ensemble de l'œuvre de l'artiste, dans le caractère d'une époque ou celui d'un peuple?

Il est impossible de trancher définitivement quant à la méthode à laquelle revient, dans la pratique, la priorité. Mais nous pouvons constater que l'on commence à l'heure actuelle à s'affranchir des seules considérations historiques qui jusqu'ici dominaient à l'excès. La même force qui pousse le philosophe à supposer que ce qui est porteur de valeur esthétique relève d'un ordre indépendant, pousse à son tour l'historien vers l'objet en tant que tel, vers la chose elle-même. Si, face à ce tournant, l'on redoute le danger d'un rationalisme insipide ou d'une réglementation abusive, c'est que l'on sousestime l'efficacité de la formation historique que nous avons tous reçue. Nous 
n'oublierons jamais que l'histoire entre par essence dans toute science de l'esprit, mais nous ne voulons plus à l'avenir servir un historisme borné.

6 Entre l'histoire de l'art et la science systématique de l'art, il y a un rapport de réciprocité qui n'autorise pas à considérer l'une des deux parties comme objectivement supérieure à l'autre, mais oblige à considérer les deux directions comme dépendantes l'une de l'autre. Quand, par exemple, l'étude des monuments désigne certains objets formés par la main humaine comme des monuments de l'art, elle doit se fonder sur un concept issu de l'art (plastique); elle n'entend pas cependant le définir a priori, mais à partir des faits que ce concept précisément a servi à circonscrire. Quand on écrit une histoire de la nouvelle, on doit savoir d'emblée ce qu'est une nouvelle; mais d'où pourrait-on abstraire un concept globalement correct pour qualifier le genre si ce n'est de l'ensemble des "nouvelles"? Ou bien pour dire les choses de manière plus générale: pour appréhender une matière historique factuelle, il faut partir de catégories systématiques que le chercheur, par souci d'exactitude entend déduire de la comparaison de plusieurs faits. Cette inévitable circularité explique ce qui a été affirmé plus haut quant à la dépendance réciproque de la pensée historique et de la pensée systématique. Nous comprenons aussi maintenant pourquoi on a d'une part reproché à toutes les catégories de genre de la science de l'art d'être des schémas vides, tout en les utilisant d'un autre côté comme des alliés essentiels, comme le fait par exemple notre philologie classique et l'exégèse de l'Ancien testament quand elle se propose d'étudier la structure de l'hymne, de la légende, de la composition du verset pour pouvoir apprécier ces différentes formes à l'intérieur de ce qu'on appelle le « Livre» de la Bible.

D'où résulte pour la science générale de l'art la tâche de déterminer le sens et la valeur de tels concepts directeurs. Pensons, pour illustrer notre raisonnement, à deux entités tout à fait différentes - à la forme musicale de la symphonie et au concept général de motif qui est utilisé, on le sait, dans tous les domaines de l'art. Si l'on veut identifier son sens en faisant ressortir de la multiplicité des phénomènesdes caractéristiques communes, on en arrive à des définitions fades ou à la conclusion décourageante qu'aucune constante ne se cache dans le changement historique. De fait, on ne trouvera aucun état de choses qui, se retrouvant partout, puisse contribuer à expliquer le particulier. Mais la symphonie signifie bien qu'il y a des lois, une règle qui préside à la combinaison d'idées musicales, un principe de déroulement. De même, le motif n'est pas quelque chose qui puisse être indiqué objectivement, que l'on puisse identifier en accumulant les cas d'observation; même les motifs qui ont un contenu, comme l'image de la descente de croix ou le motif poétique de l'inceste sont des principes de relation, des forces qui organisent et qui donnent forme, des indicateurs d'une certaine organisation de l'espace, des repères dans la construction du drame. Si jusque-là les motifs étaient mal compris, soit qu'on les considérât sur un plan psychique ou intellectuel en tant qu'idées, soit qu'ils prissent un caractère objectif en tant que noyau ou centre d'un ensemble, il faudrait désormais leur attribuer une fonction esthétique.

8 Car ce tournant - qui vient après un tournant déjà achevé dans la logique - permet de conserver toute sa valeur à la particularité de tout phénomène historique. Le fait singulier n'est plus pressuré pour qu'en soit extrait un concept général creux, il est conservé dans toute sa plénitude comme l'indispensable secours qui soutient le pouvoir de la règle générale. Si une œuvre d'art entre dans une certaine catégorie, cela signifie qu'elle constitue une étape dans un processus d'instauration de lois; en cela, sa signification ne s'épuise pas dans l'appartenance à une règle telle qu'elle peut être 
décrite par la science systématique de l'art, mais réside tout autant dans la particularité historique qui en tant que telle assigne à cette configuration [Gebilde] particulière sa position au sein des multiples possibilités de réalisation du principe. De la même manière que la constance de l'idée de droit n'invalide pas les particularités de la législation historique, les règles de la science de l'art ne font pas non plus apparaitre la particularité des styles comme secondaire. Ce qu'une époque, un peuple, un homme a pu proclamer de plus profond et de meilleur ne disparaît jamais, de la même manière que la variété des lumières multicolores qui nous entourent ne disparait jamais bien qu'elles obéissent à l'unique loi de la réfraction. Il s'ensuit qu'une théorie bien pensée des concepts de la science de l'art ne peut être féconde que si elle se réfère en permanence à la connaissance historique, tandis que cette dernière ne peut l'être que grâce à la systématique.

9 Aujourd'hui, le fructueux travail d'une psychologie devenue indépendante fait partie de la systématique. La psychologie moderne a étudié la perception [Aufnehmen] esthétique et la création artistique, soit en considérant le processus comme une entité unique soit en le divisant en différentes composantes ou en différentes phases temporelles. Mais le plus souvent, la relation qu'entretient le processus subjectif avec la constitution artistique [die kunstvolle Verfassung] stricte de l'objet restait non élucidée. Quelques éclaircissements sur la question furent apportés par les expériences menées à partir de transformations arbitraires, par exemple en considérant une figure d'abord seulement dans ses proportions, puis en tenant compte des forces à l'œuvre dans les formes pour constater que cela donnait lieu à des jugements esthétiques tout à fait différents. D'autres travaux de psychologie furent plus importants encore; ils partaient de rapports mesurables de lignes, de mesures musicales, de couleurs pour distinguer des rapports déplaisants, indifférents et plaisants, ou ils visaient à la simplification des objets artistiques pour atteindre à une configuration qui autorisait une pratique expérimentale. Il apparut alors de plus en plus clairement que les objets ne sont pas des stimuli indifférents déclenchant un état psychique, mais qu'ils sont porteurs de valeurs esthétiques. Quand le psychologue, dans ses expériences, produit un objet d'expérimentation, cela doit se faire avec un jugement esthétique, car l'objet est plus qu'un simple objet de stimulation vide. Si la science de l'art continue dans cette voie, elle comprendra qu'elle se doit d'explorer la structure de formes spatiales, de compositions rythmiques et autres dans la mesure où elle relève d'un vouloir artistique [Kunstwollen] et conduit à une attitude artistique. Une telle étude ne traite naturellement pas d'une configuration spatiale [Raumgebilde] dans le sens de la géométrie, mais comme une chose objective, c'est-à-dire comme un ensemble de lois autonome ou comme un tout avec des règles structurelles démontrables auxquelles est lié l'effet esthétique. Notre science générale de l'art se propose d'établir à l'aide de concepts fonctionnels une théorie de la structure des objets esthétiques et, dans ce propos, elle retrouve l'esthétique philosophique et psychologique. C'est le premier profit à tirer de notre réflexion.

10 Mais dès lors, on ne peut plus éluder la question de la position passée, présente et future adoptée vis-à-vis de notre propos par la recherche dans le domaine de l'art plastique, de la littérature et de la musique. Il faut rappeler que les historiens des arts furent longtemps empreints de la méfiance la plus profonde quant à toute forme d'esthétisation. Ils ne voulaient pas sacrifier leurs conclusions exactes au goût personnel ou aux caprices des modes ; ils craignaient que les faits ne soient déformés par le bavardage des philosophes. Il pensaient avoir érigé à travers la recherche 
historique un solide bastion contre les déferlements de belles paroles. Ils en oublièrent totalement que la spécificité de l'objet artistique interdit de simplement reprendre les méthodes qui avaient fait leurs preuves sur les processus politiques et les constitutions des États. Ils commirent une faute comparable à celle de leur prédécesseurs en philosophie: si ces derniers, séduits par l'intellectualité parlante des phénomènes esthétiques, avaient exclusivement apprécié la teneur des œuvres, les historiens "seulement historiens ", convaincus de l'importance de la tradition écrite, accordèrent leur attention à leur contenu et à leur sujet. Ils se souciaient peu du fait que, par exemple, tout tableau est un morceau de peinture, toute colonne un morceau de sculpture, que les tableaux, les statues et les édifices relèvent d'un archétype de formes particulières d'expression, d'activités et d'ouvrages traditionnels, voire de tâches techniques. En vérité, la teneur et le sujet ne doivent pas être saisis à travers l'apparence visible, c'est en se plongeant dans la forme artistique que l'intérieur doit en fait être saisi. Dans l'histoire de la littérature, l'étude critique habituelle des sources commet la même erreur, car ce qui importe ce n'est pas le sujet, ni non plus les modifications de contenu par rapport à la tradition, mais la mise en forme [Gestaltung]. Bref, il ne devrait pas y avoir d'historiens de l'art aveugles aux impressions du tableau, ni d'historiens de la littérature non réceptifs aux formes du langage, ni d'historiens de la musique indifférents à l'univers fabuleux des sons.

11 Mais même quand la compréhension de la matière historique est portée par une connaissance générale des conditions de vie de l'art, ce n'est pas encore une condition suffisante pour résoudre le problème. Au contraire, ce qui est véritablement crucial, c'est ce que l'on considère - soit en général, soit pour une période donnée - comme l'essentiel d'une forme particulière d'art. Si, par exemple, on croit qu'en peinture, c'est la couleur qui constitue la valeur essentielle, et donc que tous les problèmes picturaux sont des problèmes de couleur, le critère pour organiser et juger les faits historiques est autre que si, avec Alois Riegl, on ne considère la couleur que comme un simple moyen utilitaire permettant de représenter des morceaux d'espace (choses) limités et des espaces illimités. En outre, toute idée plus précise de la spécificité d'une forme d'art est liée à l'idée d'un idéal vers lequel tend l'évolution de cette forme d'art. Un tel idéal apparaissant comme fin et aboutissement d'un progrès constant, on a certes une historiographie commandée par une systématique, mais cependant pas tout à fait mature.

12 Les manques auxquels je pense sont manifestes là où un style particulier du passé est considéré comme la norme générale, indépassable. Les choses sont déjà plus difficiles quand la fin naturelle à un moment donné est spontanément tenue pour l'accomplissement du processus souhaité. Ainsi, l'esthétique des Lumières, dans sa belle assurance, n'a saisi dans l'art ancien que ce qui pouvait passer pour une étape vers sa propre vision; et l'« Histoire générale de la musique » de [Johann Nikolaus] Forkel (1788-1801) est un dernier exemple de cette manière linéaire de considérer l'évolution artistique. Et de nos jours encore, on écrit ça et là l'histoire pour exalter une certaine orientation de la création contemporaine dans laquelle on voit un achèvement.

13 En face, d'autres historiens ont mis en lumière la valeur propre de modalités artistiques différentes selon le temps et les nations. Empreints d'un esprit de justice sans préjugés, ces derniers déclarent que toute chose, là où elle se situe dans l'histoire, a une valeur, remplaçant le concept de péremption par celui de transition. Des chercheurs se joignent à eux qui enseignent une succession typique de courants - voyant par 
exemple, dans l'évolution de l'histoire de l'art, des périodes au cours desquelles c'est la forme constructive qui est recherchée, d'autres pour lesquelles c'est la forme expressive qui est considérée comme l'idéal, et d'autres en revanche encore où c'est l'idée de l'imitation de la nature qui préside à la création. Une fois ces possibilités épuisées, le jeu recommence, le plus souvent de manière accélérée. De telles constructions artificielles trouvent une certaine justification quand il s'agit de décrire un problème particulier. Si l'on examine des œuvres d'art, peu importe lesquelles, à partir d'un seul aspect, elles sont plus faciles à ranger dans une succession objectivement nécessaire de tentatives de solution à un problème artistique donné. Mais il est difficile de réussir à contraindre la complexité d'authentiques configurations [Gebilde] artistiques dans le carcan d'un concept de genre préétabli. De même, en violant le sujet par exagération ou déformation, ou par négligence, on fera disparaître la belle vitalité et la mobilité de l'histoire.

Dans le processus que nous venons de décrire, la foi en une conceptualité rigide et substantielle semble jouer un rôle. Les historiens modernes de la littérature appuient parfois leur description sur des concepts généraux solides. Une histoire de l'ornement musical récemment publiée commence par une analyse formelle qui rappelle la logique hégélienne avant de retrouver les regroupements ainsi découverts dans le devenir historique de la musique. Aussi louable que soit la tentative de dépasser philosophiquement le caractère fortuit de l'existence historique à travers des relations conceptuelles, on n'a sans doute pas encore réussi jusqu'ici à fondre véritablement les catégories logiques avec l'incommensurable richesse d'une réalité historique versatile qui, dans les détails, change en permanence. Il sera nécessaire d'appliquer le mode d'observation déjà recommandé qui repose sur l'hypothèse d'une nécessaire unité intellectuelle s'exprimant sous des formes différentes. Cette unité, cependant, n'existe pas pour elle-même ; elle se manifeste exclusivement dans le fait que, raisonnablement, une période n'est pas possible sans une autre, que chaque style recèle un aspect d'une époque antérieure et d'une époque à venir. Les relations qu'elles entretiennent reposent sur ces références mutuelles; on pourra comparer les valeurs spécifiques particulières en considérant les forces qui sont à l'œuvre en elles comme les différentes possibilités d'expression d'une même loi.

En examinant de ce point de vue les tendances historiques actuelles dans trois domaines principaux, nous observons partout les signes avant-coureurs d'une alliance naturelle avec la science systématique de l'art, mais guère plus, il est vrai, que les indices de ce qu'on peut espérer de l'avenir. La méthode la plus simple et donc la plus répandue est celle du biographisme. Certes, il est sûrement intéressant d'apprendre quel fut le destin de tel ou tel artiste, quels prédécesseurs il a vénérés, quel enseignement il a reçu, quels buts il a sciemment poursuivis. Mais il y a cependant, dans l'usage qu'on en fait ordinairement, de grotesques malentendus. Le simple fait de mettre la création sur le même plan que, par exemple, les conceptions exprimées par l'artiste suffit à semer le doute : on ne rend pas compte de l'art de Dürer en l'expliquant à partir de ses principes scientifiques. L'abondance de témoignages et de théories énoncés par des écrivains dont dispose l'histoire de la littérature lui a été fatale, car le plus souvent on ne voit pas que dans les formes qu'un poète a produites, sont à l'œuvre des forces dont il ne sait rien lui-même ou qu'il méconnaît. Plus dangereuse encore, la folie qui consiste à voir dans la matière première qu'est la vie un infaillible moyen d'explication. Une certaine recherche philologique consacrée à Goethe règle son compte au psychisme d'Oreste et de Pylade en renvoyant à deux phases de l'évolution 
du poète, ne voyant dans Iphigénie qu'une image de Charlotte von Stein, transfigurée par l'idéal féminin de Goethe, en pensant apparemment pouvoir s'affranchir d'autres devoirs vis-à-vis de l'œuvre. Il est aussi arrivé que quelqu'un entreprenne de dégager des chants d'un poète de la même époque toutes sortes de données biographiques; confronté à la démonstration de ses erreurs, il répliqua que ces conclusions erronées n'étaient qu'une preuve de plus du « manque de clarté » du poète!

Pour être juste, il faut rapidement ajouter que [Wilhelm] Scherer, dont se réclament volontiers ces égarés, s'est montré plus clairvoyant. Certes, lui aussi privilégiait les éléments biographiques et les sources du poète, mais il soulignait toujours avec une belle insistance l'altérité de la forme artistique ; son erreur fut de penser qu'on pouvait dégager l'âme artistique de l'œuvre en soustrayant simplement le vécu et l'acquis. Mais au sein de l'école de Scherer, la réflexion malgré tout considérable du maitre s'est souvent trouvée recouverte par une absurde mécanisation; la foi en la vérité d'un modèle et l'attachement à la date inutile caractérisent ces enthousiastes du savoir superflu. Il a fallu qu'intervienne la psychologie, sur laquelle se portaient à l'époque les plus grands espoirs, pour que quelque changement apparaisse. On vit apparaître de brillants " caractérisateurs " pour lesquels toutes les dissections et toutes les études stylistiques d'une œuvre servaient en fin de compte à découvrir l'homme derrière l'artiste. D'autres entamèrent leur travail en ayant déjà au cœur une image d'ensemble de la personnalité du poète, se plongèrent dans le sentiment Qu'il avait de sa vie au moment de la conception artistique, et explorèrent comment la personnalité et ce sentiment inscrit dans le temps pouvaient modeler la matière de l'œuvre jusqu'à sa plus subtile expression. On pensait toujours secrètement que, dans le devenir, on ne saisissait rien d'autre que l'essence. Quant à nous, nous réclamons que l'on dépasse ce psychologisme, que l'on parte objectivement de la composition et de sa forme linguistique pour n'observer l'évolution de l'œuvre qu'en complément; nous constatons avec bonheur que dans les livres sur Shakespeare et l'esprit allemand, sur "Vérité et poésie ", sur le romantisme, la construction artistique des œuvres est prise en compte avec délicatesse et attention.

Voici ce qu'il en est de la référence aux dépendances personnelles, aux motifs inspirés par autre chose et aux moyens techniques. Le même sujet et la même mise en forme peuvent être issus de positions artistiques fort différentes. Il faut une sensibilité bien entraînée et un esprit philosophique perspicace pour saisir le processus essentiel sans se laisser aveugler par la ressemblance des symptômes. L'affinité de deux artistes ne se dévoile que si, partant de l'apparence des similitudes extérieures, on pénètre au plus profond des œuvres pour conclure qu'un homme ou son art a pu être ressenti, accueilli intérieurement et, en cela, transformé selon certaines lois par un autre. Un sculpteur vivant d'aujourd'hui peut être plus proche de la statuaire grecque qu'un copiste romain. Nous ne cherchons pas les élèves, mais les généalogies [Blutzeugen]. Même le retour de tout un style ne signifie pas une simple répétition, c'est la réapparition d'une position que la conscience adopte pour de nouvelles raisons et à de nouvelles fins par rapport à la réalité.

Ces restrictions valent pour l'historiographie de tous les domaines de l'art. Pour l'histoire de la musique par exemple, elles portent sur ce qu'on appelle les réminiscences. Quand Beethoven élève un joyeux thème mozartien jusqu'à en faire l'idée principale de sa Symphonie Hérö̈que, cela ne s'explique véritablement qu'à travers le processus que nous venons d'évoquer. L'exégèse volontiers pratiquée aujourd'hui 
trouve entre des morceaux de musique et des événements de la vie des relations qui sont problématiques ne serait-ce que parce que le moi civil et le moi artistique ne coïncident jamais. De plus, elle se méprend en décrivant la valeur psychique comme étant le noyau des différentes formes sonores à partir des concepts indigents que notre psychologie vulgaire emploie pour les affects. On admire parfois la virtuosité littéraire de la description, mais on regrette souvent que cette analyse ne pénètre pas jusque dans les relations temporelles, dynamiques et qualitatives des sentiments - sans même parler du fait qu'elle néglige la forme.

Si nous regardons en arrière, le biographisme se présente sous deux formes, une forme extérieure et une forme psychologique. Mais il peut aussi prendre une forme plus large quand l'individualité de l'artiste est comprise comme un produit de toute son époque. On n'aurait guère besoin de parler ici de cette forme d'observation relevant de l'histoire de la culture si elle ne comportait pas en soi une prémisse systématique tout à fait particulière. En effet, dès que l'individu est assimilé à son peuple et à son temps, l'attention de l'historien se concentre sur le contenu de l'œuvre. Il faut lire comment Springer traite le portrait de Jules II par Raphaël, ou comment Janitschek démontre que Dürer a transposé toutes les inspirations étrangères, c'est-à-dire étrangères sur le plan du sujet, en quelque chose de germanique parce que «les formes de vie dépendent, comme les idéaux, du sol et de l'air... ». C'est Karl Justi qui a pratiqué le procédé de la manière la plus subtile, en cherchant à expliquer l'œuvre dans le contexte des conditions politiques, économiques et intellectuelles de la situation culturelle, certes non pas directement, ou quasi arithmétiquement, mais bien en expliquant que l'œuvre se détache d'un fond commun d'activité. Dans cette vision historique, il oppose au dogme de la valeur exclusive du «comment» en art, la théorie de la signification du «quoi ».

Or, il me semble que toutes ces manières de traiter le sujet ne touchent au fond que la périphérie de l'œuvre d'art: elles s'attachent à l'œuvre d'une certaine manière rétrospective, guettant tout ce qui fut son milieu, tâtonnant autour de ce que circonscrit l'histoire de la culture mais en restant toujours en dehors du centre, en dehors de la chose elle-même. Pour dire la vérité, peu de gens sont conscients de cette relation. Si, malgré tout, à côté de cette théorie de la périphérie, une science des choses s'est développée, c'est en partie pour des raisons extérieures. Lorsque, par exemple, l'archéologie s'est enrichie de découvertes que n'accompagnait aucun témoignage écrit, la dissection stylistique était bien obligée de remplacer toutes les autres méthodes, il arriva alors assez souvent qu'à partir de l'étude stylistique de l'objet, on ait tiré des conclusions quant à son auteur. La connaissance de l'art préhistorique dépendait et dépend encore complètement de l'explication objective et de la recherche sur la forme. L'étude attachée directement à l'objet s'est développée seule, en dehors d'une telle contrainte des faits. On peut saluer avec joie ce qu'elle se propose de faire, sa méthode n'a pas besoin d'être défendue.

21 L'exemple le plus curieux d'application erronée d'une idée juste se trouve dans les écrits de Lermolieff. Lermolieff voulait attribuer à un artiste des tableaux difficilement identifiables en étudiant les lois de son style personnel considéré comme immuable. Ce style, il le trouvait dans la forme de la main, du nez de l'oreille, du crâne, des rides. L'impression d'ensemble de l'œuvre (même en relation avec des témoignages écrits) ne suffisait pas, selon lui, pour reconnaître d'emblée le peintre; au contraire, il fallait avoir une connaissance des formes particulières au grand maittre pour pouvoir 
distinguer un original d'une copie ou d'une œuvre d'école. Subrepticement, chaque toile devenait ainsi pour le critique une collection de détails marqués par une individualité. C'est l'esprit d'une science ancienne de la nature qui triomphait ici, le même que celui qui avait poussé Semper à voir dans l'œuvre d'art un produit mécanique composé d'une valeur d'usage, de matière première et de technique. Peu à peu, la conception qui voyait dans l'œuvre un composite glissait vers d'autres facteurs et on voyait apparaître une école aux activités multiples. Beaucoup de chercheurs travaillent dans le sens de cette érudition artistique parce que, outre un certain goût, susceptible aussi de s'éduquer, elle ne réclame que de résoudre un problème d'ordre mécanique. L'accumulation de cette sorte de travaux donne l'impression d'une discipline assurée. Mais pour des exigences plus élevées, il faut une autre qualité de talent et aussi la conviction, modeste, qu'une authentique œuvre d'art est une création et non pas une somme arithmétique. Ce fut, on le comprend aisément, sur l'instigation de certains cercles d'artistes que quelques historiens encore vivants prirent catégoriquement position contre le matérialisme en art et le caractère unilatéral de la critique du style. Bien que la nouvelle génération commence maintenant à les suivre, la masse des historiens d'âge moyen reste attachée aux préalables que nous avons critiqués.

De la même manière, si l'on parcourt les travaux de philologie objective, le résultat est tout aussi peu réjouissant. Dans la plupart des textes s'intéressant aux questions de formes et de styles, règne un atomisme rationaliste. Quelqu'un veut-il décrire la beauté formelle de la Bible à partir d'images et de comparaisons. Comment s'y prend-il ? Il compte les animaux qui y figurent, commence par le bétail pour finir par la puce et l'escargot. Un philologue travaillant sur Goethe étudie les hyperboles dans Götz, en l'occurrence en les classant en «a) ciel et enfer, b) grands nombres, c) autres hyperboles »- une méthode d'une superficialité et d'une stérilité inégalables. Car s'il est clair que l'art poétique doit être compris à partir du moyen dont il se sert pour produire ses effets, le langage, il est tout aussi clair que c'est la tonalité d'ensemble du tout, la force poético-linguistique, qui doivent d'abord être saisis en tant que tels. Il relève de la plus fâcheuse esthétique de tiroirs que d'entreprendre d'étudier si le poète "représente le passé ou l'intemporel ou l'avenir », que d'oser décréter que "le verbe est plus poétique que le nom, le nom plus poétique que le pronom». Je reconnais volontiers que de telles conceptions sont pour moi contraires à l'art et m'apparaissent de ce fait comme sans valeur du point de vue de la science de l'art.

Il ne faudrait néanmoins pas donner l'impression erronée que le courant que je défends ici est opposé à la bonne vieille philologie. Nous apprécions sa capacité à dissiper l'étrangeté de ce qui est ancien, à dégager les formes d'art et le bénéfice intellectuel d'une littérature de leur enveloppe extérieure; nous rendons hommage au sentiment artistique que peut recéler le silencieux travail de débroussaillage du texte. Personne parmi nous ne refusera son admiration à des hommes comme Lachmann et Müllenhoff; d'autant moins que leur tentative d'étudier, à travers une critique supérieure, le devenir progressif des constructions littéraires, partait de la chose même. En démontrant des incohérences dans la narration de l'Iliade, des différences de style et de valeur, et en particulier un étonnant changement de ton et de couleur dans la description, Lachmann a soumis l'œuvre à un examen et à un nettoyage véritablement esthétiques. Mais malheureusement les catégories esthétiques de Lachmann et de Müllenhoff étaient insuffisantes. Leur théorie globale, leur idée de chants « additionnés ", « organisés », « retravaillés par la poésie » dut être abandonnée parce 
qu'elle était mécanique. La recherche récente repose sur une meilleure connaissance de l'essence de l'épopée, non pas du poème épique en ce qu'il est construit, mais tel qu'on en a observé l'influence historique; elle montre que le corps épique, constitué dans de tout autres relations structurelles, ne peut naître de l'addition de formes en soi parfaitement cohérentes. Aujourd'hui, la résistance si vive contre la formule traditionnelle qui veut que la légende héroïque soit un mélange de mythe et d'histoire m'apparaît comme une réponse à la vision mécaniste d'une part, au mode de pensée rationaliste d'autre part. La libre poésie, reconnait-on aujourd'hui, forme également l'essence de la légende germanique ; c'est à partir de tout l'organisme vivant qu'on juge désormais la relation au mythe et à l'histoire qui est seulement possible comme inhérente à la légende telle qu'elle est faite.

En suivant encore plus avant les lignes de faille du nouveau mouvement, nous trouvons d'abord une étude des poèmes de Walther von der Vogelweide qui pose à la poésie lyrique médiévale des questions jamais soulevées auparavant avec autant de clairvoyance et de clarté. Au lieu de dater selon le sujet et la matière et d'assigner à la jeunesse ce qu'on appelle les chants du «menu amour» [niedere Minne], et ceux de l'«amour noble » [hohe Minne] à l'âge mûr du poète, c'est par comparaison avec le style de son professeur Reinmar que celui de Walther est étudié ; l'idée directrice, salutaire, est que tout jeune poète suit un maître : les poèmes qui suivent la manière de cour fade de Reinmar sont attribués à la première période à partir de subtiles considérations stylistiques et métriques, tandis que ceux qui sont plus proches de la poésie populaire des chevaliers et des vagants le sont à la période où Walther, désormais poète itinérant, n'est plus à la cour. Ainsi, le chercheur en quête de l'esprit d'une œuvre en arrive-t-il à élucider jusqu'aux mystères de la datation. Un autre exemple peut être mentionné dans la philologie classique. Les œuvres de Sapho que nous connaissions jusque-là viennent d'être complétées par un poème de clair de lune qui invite à la comparaison avec Goethe; par empathie avec l'âme de la femme, à travers une contemplation pleine de sensibilité du ciel étoilé du sud, non seulement ce petit bijou nous est rendu accessible, mais de plus, on voit se dégager une loi objective de la force d'illustration et de composition de l'art. Enfin, il faudrait penser à la théorie des constantes sonores en poésie et en musique. Sa dernière condition préalable serait en effet de reconnaître que la vie propre de l'œuvre d'art comporte un certain nombre d'exigences vis-à-vis de celui qui l'accueille, exigences que ce dernier, pour peu qu'il soit assez sensible et ne soit pas inhibé par la convention, remplit sans le vouloir. On rompt ici avec ce qu'on admet généralement, à savoir que l'on pourrait lire les vers comme on l'entend, chanter une phrase mélodique de Wagner comme une cantilène de Bellini; on affirme ici avec plus de profondeur qu'une composition artistique n'est achevée qu'à partir du moment où quelqu'un en jouit, car cette jouissance n'est pas quelque chose qui varie arbitrairement, elle est la satisfaction de la volonté d'art incarnée dans l'objet, et en cela obéit à des lois.

Quelques brèves remarques devraient alors suffire pour faire comprendre la différence entre la foi nouvelle et la foi ancienne en ce qui concerne le domaine de l'art plastique. Tandis qu'autrefois les styles étaient classés d'après les formes singulières ou même les ornements, c'est maintenant dans l'ensemble de la vision artistique que l'on cherche l'essence d'un style. Ce ne sont pas les arcs en plein cintre qui font d'un monument un monument roman, mais les lignes directrices de la mesure de l'espace, les forces dynamiques à l'œuvre dans son articulation, enfin les intentions - et les proportions qui en découlent - présidant à la construction conçue comme un tout. Une statue ne se 
compose plus des caractéristiques de son apparence, elle doit être saisie à partir des lois de la forme qui la rendent possible comme l'expression fonctionnelle d'une conformité objective à des règles. Le même passage de la partie au tout, de l'extérieur à l'intérieur semble également s'accomplir dans la musicologie. Mais il nous faut (et nous le pouvons) renoncer à entrer dans une démonstration de détail.

Arrivés à ce point, on voit désormais se dégager une magnifique perspective. Nous apercevons les vastes terres vierges que pourra exploiter la recherche. Que l'on vienne de la philosophie et de la psychologie, ou de la recherche historique, c'est toujours vers le même centre que l'on se voit dirigé. Quand on entre dans notre domaine à partir de l'une de ces directions, on doit reconnaître que les objets esthétiques et à plus forte raison les objets de l'art ne tirent pas leur spécificité de l'attention qu'on veut bien leur prêter, mais de règles du jeu qui leur sont inhérentes. Mais l'historien ne doit surestimer ni les phénomènes secondaires de la biographie ni ceux de l'histoire culturelle, il doit, avec l'essence de la chose, saisir son devenir, avec la composition objective son environnement. C'est ainsi que la représentation historique peut devenir une esthétique appliquée : qu'elle reste de l'histoire, mais en se nourrissant de la valeur propre de l'art. Aucun spécialiste ne peut désirer la mort du sens historique; nous ne faisons que revendiquer un peu plus de marge de manœuvre pour la science qui s'attache à l'œuvre elle-même, car les concepts semi-inconscients d'un esprit non systématique ont généralement une utilité limitée au seul usage domestique de leur inventeur.

La science générale de l'art explore la constitution des objets sous un double point de vue : elle relève d'une volonté d'art et elle est destinée à une jouissance artistique ; cette théorie structurelle part du tout dont elle suit l'articulation et dont elle trouve l'unité dans une organisation fonctionnelle de valeurs combinatoires. Elle justifie la particularité d'une apparition particulière comme étant un degré dans les lois qui commandent au processus et se déploient tout autant dans le rythme général du mouvement de l'art que dans l'objet idéal. L'unité intellectuelle de l'évolution historique réside elle aussi dans un ordre fonctionnel de valeurs combinatoires, dans la loi qui veut qu'un style renvoie à un autre. Ce qu'il ne faut pas confondre avec le préalable habituel de l'histoire qui procède par problèmes et qui consiste à toujours partir d'une visée objective à atteindre. Car ce qui induit que des états de faits historiques sont des grandeurs comparables, c'est la règle selon laquelle ils renvoient les uns aux autres.

\section{NOTES}

1. Cet exposé, qui s'inscrit dans la ligne d'articles plus anciens publiés ici sur «Scepticisme en esthétique » et « Objectivisme en esthétique » est le texte très peu remanié du discours prononcé par l'auteur à l'occasion de l'ouverture du Congrès de Berlin le 7 octobre 1913.

L'auteur ne citant pas ses sources, il n'a pas été possible de les préciser dans la traduction (NDT). 
INDEX

Mots-clés : philosophie, catégories esthétiques, histoire de l'art, systématique de l'art, style Schlüsselwörter : Philosophie, Kunstgeschichte, ästhetische Kategorien, Systematik der Künste, Stil

\section{AUTEURS}

\section{MAX DESSOIR}

Philosophe. (Plus d'informations ici) 